\title{
International Congress Programme Committee Core Group members
}

Dr. Annie SUGIER (ICPC Chairperson)

Institut de Radioprotection et de Sûreté Nucléaire, France

Dr. Leopoldo ARRANZ (IRPA Vice-President for Congress Affairs)

Hospital Ramón y Cajal, Spain

Prof. Eduardo GALLEGO (ICPC Scientific Secretary)

Universidad Politécnica Madrid, Spain

Dr. Jacques LOMBARD (ICPC Chairperson Assistant)

Société Française de Radioprotection, France

Dr. André BOUVILLE

National Cancer Institute, USA

Dr. John COOPER

National Radiological Protection Board, United Kingdom

Dr. Bobby CORBETT

Hairmyres Hospital, United Kingdom

Dr. Renate CZARWINSKI

Federal Office for Radiation Protection, Germany

Dr. Antonio DELGADO

Centro de Investigaciones Energéticas, Medioambientales y Tecnológicas, Spain

Dr. Rick JONES

U.S. Department of Energy, USA

Dr. Juan Carlos LENTIJO

Consejo de Seguridad Nuclear, Spain

Dr. Sigurdur MAGNUSSON

Icelandic Radiation Protection Institute, Iceland

Dr. Ches MASON

International Atomic Energy Agency, Austria

Prof. Henri MÉTIVIER

Institut de Radioprotection et de Sûreté Nucléaire, France

Dr. Alastair MCKINLAY

National Radiological Protection Board, United Kingdom

Prof. Xavier ORTEGA

Universitat Politècnica de Catalunya, Spain

Dr. Eliseo VAÑo

Universidad Complutense de Madrid, Spain 
This page intentionally left blank 